\title{
MICRO CORIOLIS MASS FLOW SENSOR WITH INTEGRATED CAPACITIVE READOUT
}

\author{
J. Haneveld, T.S.J. Lammerink, M.J. de Boer and R.J. Wiegerink. \\ MESA+ and IMPACT Research Institutes, University of Twente, The Netherlands. \\ e-mail: j.haneveld@utwente.nl
}

\begin{abstract}
We have realized a micromachined micro Coriolis mass flow sensor with integrated capacitive readout to detect the extremely small Coriolis vibration of the sensor tube. A special comb-like detection electrode design eliminates the need for multiple metal layers and sacrificial layer etching methods. Using differential readout signals significantly reduces the influence of parasitic capacitances. In addition, the sensing electrodes have been realized on a suspended tube structure which will allow for tuning of the electrode separation by a DC current. First measurements using water, ethanol and white gas indicate that true mass flow is measured by the sensor and that sensor output is linear with mass flow. The measurement error is currently in the order of $2 \%$ of the full scale of $1.2 \mathrm{ml} / \mathrm{hr}$ for all measured liquids (which corresponds to $1.2 \mathrm{~g} / \mathrm{hr}$ in the case of water).
\end{abstract}

\section{INTRODUCTION}

Integrated microfluidic systems have gained interest in recent years for many applications including (bio)chemical, medical, automotive, and industrial devices. A major reason is the need for accurate, reliable, and cost-effective liquid and gas handling systems with increasing complexity and reduced size. In these systems, flow sensors are generally one of the key components.

Most MEMS flow sensors are based on a thermal measurement principle. It has been demonstrated [1,2] that such sensors are capable of measuring liquid flow down to a few $\mathrm{nl} / \mathrm{min}$. These sensors require accurate measurement of very small flow-induced temperature changes. An important problem of thermal flow sensors is that the measurement is highly dependent on temperature and fluid properties like density and specific heat.

The sensor presented in this paper is based on the Coriolis force which acts on a fluid (mass) flowing in a vibrating channel. Coriolis flow meters [3-5] are mostly used for measuring large flow rates, since the relatively weak Coriolis forces are correspondingly harder to detect for small flows. In general, the signal-error ratio is very sensitive to fabrication and construction errors, as well as external influences of temperature and mechanical nature. However, an important advantage of this type of sensor is that the Coriolis force is directly proportional to the mass flow and independent of temperature, pressure, flow profile and fluid properties.

\section{OPERATING PRINCIPLE}

A Coriolis type flow sensor consists of a vibrating tube. Now consider a moving mass inside the tube. This mass is forced to change its velocity due to the externally imposed vibration. This results in Coriolis forces that can be detected. Figure 1 shows a schematic drawing of a Coriolis sensor based on a rectangular tube shape. The tube is actuated in a "torsion mode" indicated by $\omega$. A mass flow $\Phi_{m}$ inside the tube results in a Coriolis force $F_{c}$ that can be expressed by:

$$
\vec{F}_{c}=-2 L \vec{\omega} \times \vec{\Phi}_{m}
$$

Where $L$ is the length of the rectangular tube (see Figure 1). The Coriolis force induces a "flapping mode" vibration with an amplitude proportional to the mass flow.

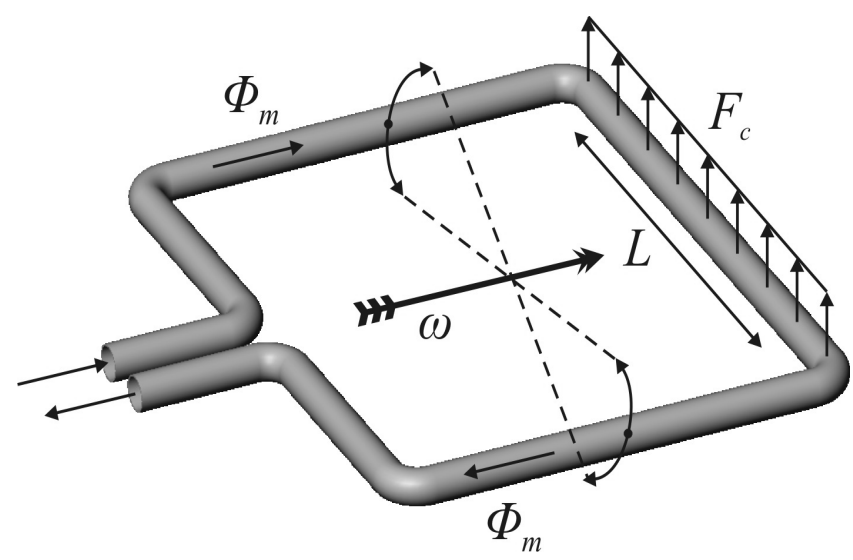

Figure 1: Rectangle-shaped Coriolis flow sensor ( $\omega$ is the torsion mode actuation vector, $F_{c}$ indicates the Coriolis force as a result of mass flow).

\section{SENSOR DESIGN}

In [6] we proposed to fabricate a micro Coriolis mass flow sensor using silicon nitride as the tube material. This resulted in a sensor with a very thin $(1.2 \mu \mathrm{m})$ tube wall, so that the mass of the tube is small compared to the mass of the moving fluid. This was a significant improvement over [7] and [8], which use silicon as the tube material, leading to a relatively heavy and stiff tube. We demonstrated that our silicon nitride sensor could reach a resolution in the order of 10 milligram/hour [6], however at that time no readout structures were integrated on the chip, so a laser vibrometer was needed to measure the outof-plane Coriolis motion of the tube.

The newly fabricated sensor incorporates capacitive readout structures, which are used to measure the very small Coriolis vibration. In addition, the fluid in- and outlets have been moved to the backside of the wafer, resulting in a more sturdy and leak-free fluidic connection. The Coriolis tube dimensions remain essentially the same: a low stress silicon-rich nitride $\left(\mathrm{Si}_{\mathrm{x}} \mathrm{N}_{\mathrm{y}}\right)$ tube with an effective diameter of approximately 
$40 \mu \mathrm{m}$ and a wall thickness of approximately $1.2 \mu \mathrm{m}$. The Coriolis tube has a rectangular shape (Figure 1) with dimensions $2.5 \times 4 \mathrm{~mm}$. Opposite the Coriolis tube is a second tube, which contains the capacitive readout structures to detect the vibration of the Coriolis tube. The detection is achieved through a comb-like structure which functions as a parallel plate capacitor when the two halves of the comb are separated out-of-plane. If necessary the tube containing the sensing structures can be moved up and down in order to tune the sensitivity of the readout structure. This is done by means of Lorentz force actuation, using a DC current. Figure 2 shows a schematic layout of the sensor, and Figure 3 is a photograph of a finished device, mounted on a PCB.

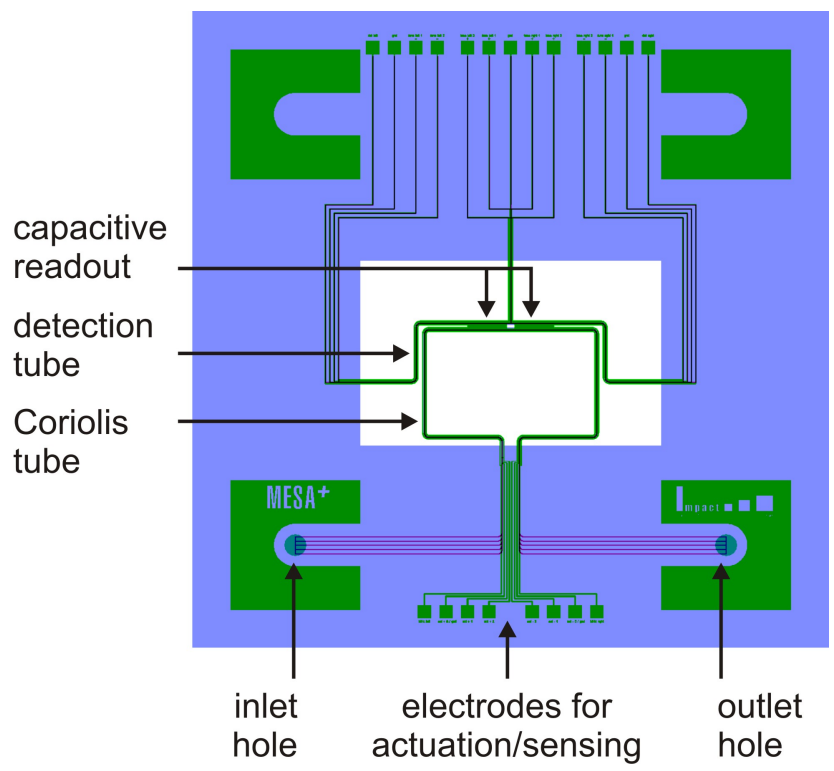

Figure 2: Layout of the sensor chip (size 15x15 mm).

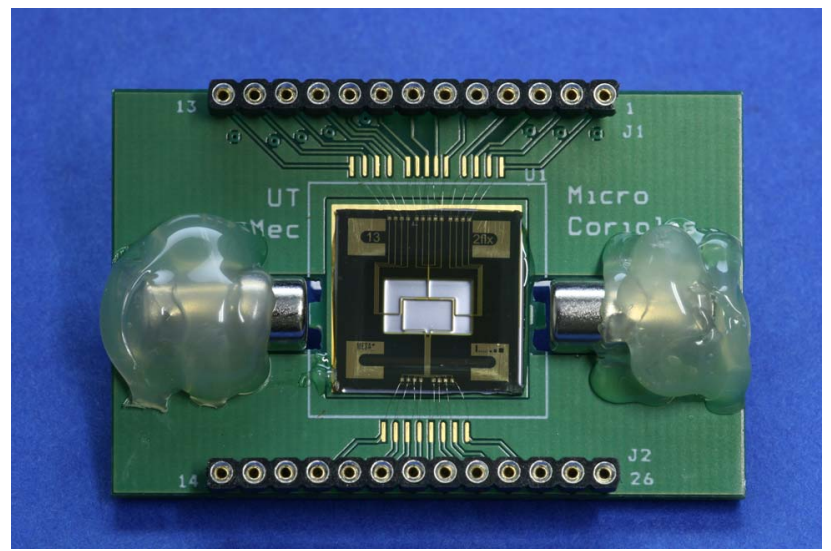

Figure 3: Photograph of mounted chip with electrical connections and permanent magnets for Lorentz force actuation. Fluidic connections are located at the backside of the $P C B$.

\section{FABRICATION}

The sensor structures were fabricated using the surface channel technology described in $[6,9,10]$ as a starting point. Figure 4 shows a summary of the fabrication process.

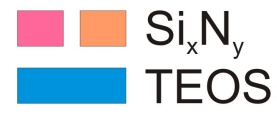

\section{a}

b

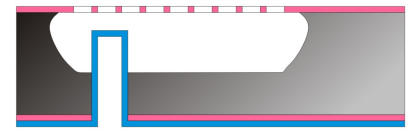

C

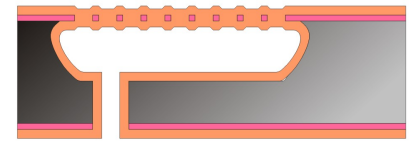

d

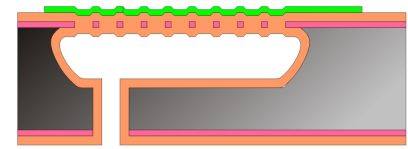

e

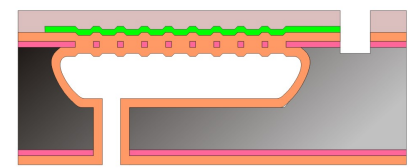

f

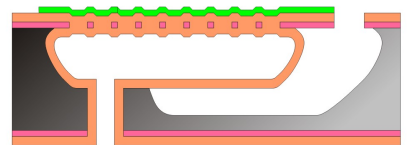

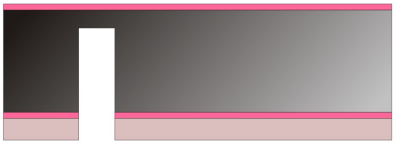

resist

$\mathrm{Cr} / \mathrm{Au}$
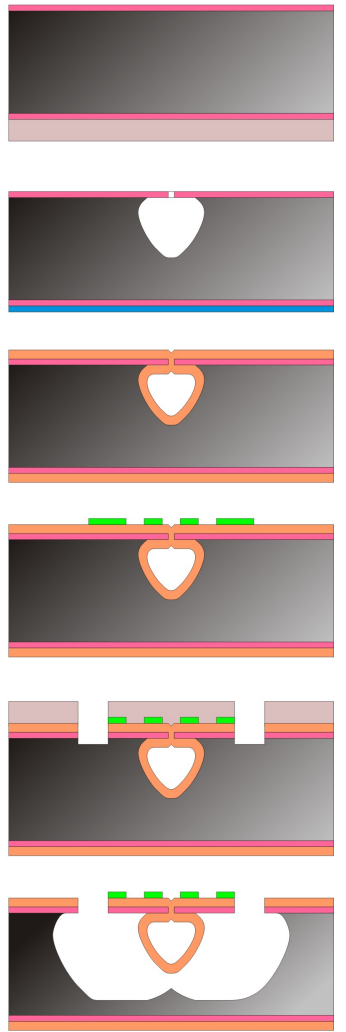

Figure 4: Outline of the fabrication process. Left column: cross-section along the length of the tube. Right column: cross-section perpendicular to the sensor tube.

Starting with a $<100>\mathrm{p}++$ wafer, a $500 \mathrm{~nm}$ thick low stress LPCVD silicon-rich nitride $\left(\mathrm{Si}_{\mathrm{x}} \mathrm{N}_{\mathrm{y}}\right)$ layer is deposited. Then fluidic access holes are etched (cryogenic pulsed $\mathrm{CHF}_{3}$ plasma etching on an Adixen AMS $100 \mathrm{SE}$ system) from the back side of the wafer. The endpoint of this etch step is aimed approximately $20 \mu \mathrm{m}$ below the top surface of the wafer and has to be carefully monitored (Figure 4a). Next a $500 \mathrm{~nm}$ thick layer of TEOS oxide is deposited on the wafer. The oxide layer is removed from the front side of the wafer using BHF etching, while protecting the oxide on the back side with a layer of adhesive dicing foil. Then a $50 \mathrm{~nm}$ layer of chromium is sputtered on the front side of the substrate. This chromium layer is subsequently patterned using a mask containing arrays of $5 \times 2 \mu \mathrm{m}$ holes, spaced $3 \mu \mathrm{m}$ apart. The pattern is then transferred to the nitride layer by means of reactive ion etching (RIE Electrotech PF 340). Then an isotropic plasma etch step is performed in an ADIXEN AMS 100 SE apparatus (Figure 4b): this defines the channel shape.

The TEOS layer and the chromium mask are then removed and another $\mathrm{Si}_{\mathrm{x}} \mathrm{N}_{\mathrm{y}}$ layer is grown with a thickness of $1.8 \mu \mathrm{m}$; this forms the channel wall and seals the etch holes in the first nitride layer in one single step (Figure 4c). A 10/200 nm layer of chromium and gold is sputtered (chromium serving as the adhesion layer for the gold) and pre-patterned using wet etching in a $\mathrm{KI} / \mathrm{I}_{2}$ etch solution, followed by a short dip in chromium etchant (Figure 4d) to create the metal tracks on top of the tube which will facilitate Lorentz force actuation and 
capacitive sensing of the structure. Then a second lithography step is performed, in which the comb-like structures are defined, as well as the windows which are used to release the tube from the substrate. The $\mathrm{Cr} / \mathrm{Au}$ layer is then patterned using ion beam etching, because wet etching makes it extremely hard to precisely control the width of the teeth of the comb structures. Directly after the metal patterning the release windows are opened by reactive ion etching of the $\mathrm{Si}_{\mathrm{x}} \mathrm{N}_{\mathrm{y}}$ layer (Figure $4 \mathrm{e}$ ). Then the structure is released by an isotropic silicon plasma etch step (using again the ADIXEN AMS $100 \mathrm{SE}$ apparatus), followed by resist removal using a Tepla 300 oxygen plasma barrel stripper (Figure 4f)).

The entire resulting structure can be seen in Figure 5 . Detailed views of the capacitive sensing structures can be seen in Figures 6 and 7.

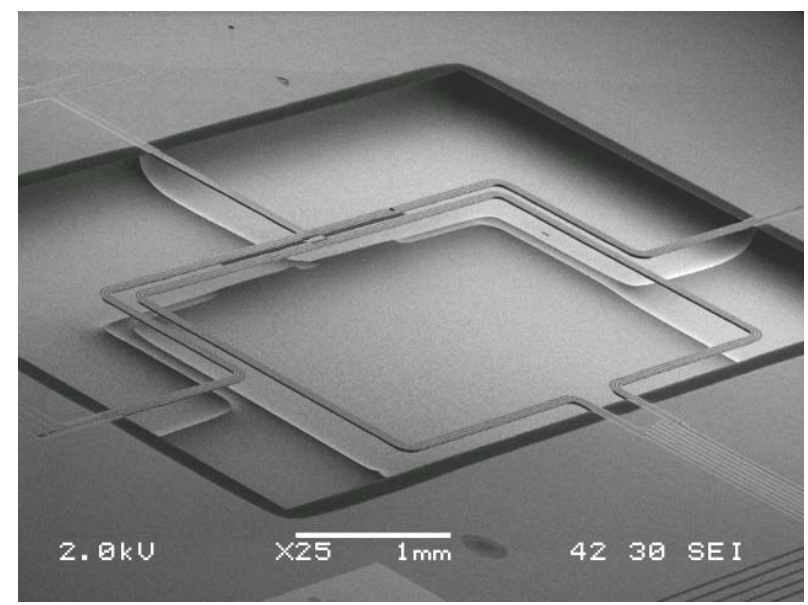

Figure 5: SEM photograph of the complete sensor chip.

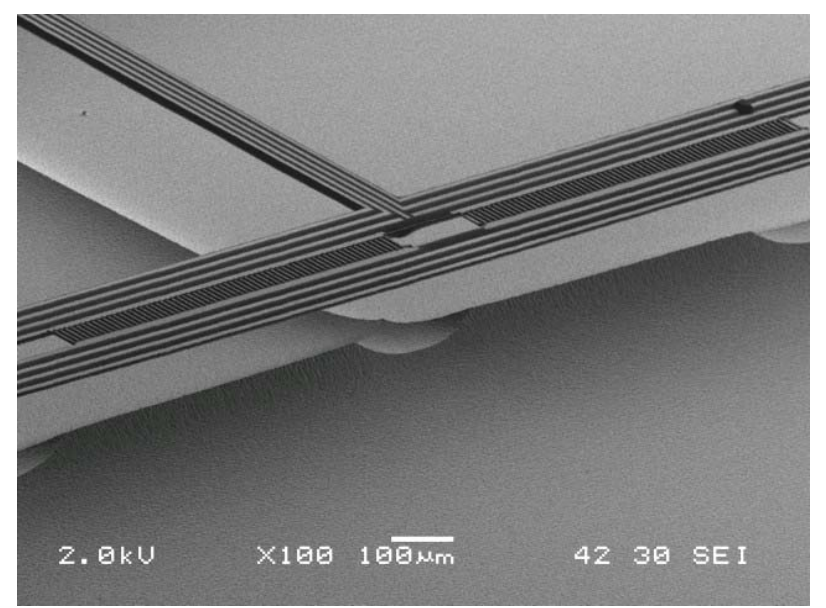

Figure 6: Close-up of the capacitive sensing structures with two comb structures to sense both the actuation and Coriolis amplitudes.

The finished chips are epoxy-glued to a PCB, which also contains pre-drilled fluidic access holes. A fluidic connector is then glued to the back of this PCB to allow for fluid/gas delivery. Permanent magnets are inserted in slots in the PCB, and permanently fixed using thermal glue. Electrical connections are made by wirebonding from the bondpads on the chip to the PCB.

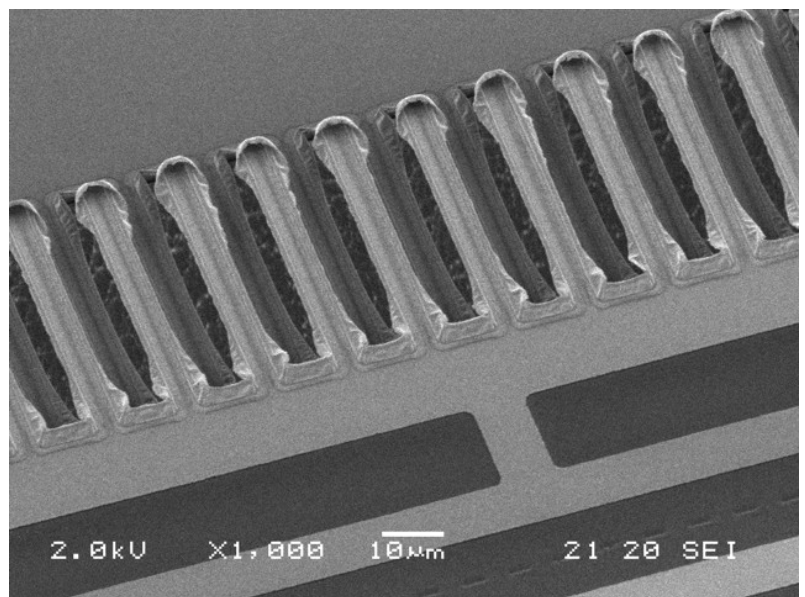

Figure 7: Detailed view of one of the comb-like capacitor structures. The lower structure is the Coriolis tube. In this case the other comb structure is attached to the bulk of the chip.

\section{MEASUREMENTS}

Mass flow measurements were done using water, ethanol and white gas. The sensor was connected to electronics which deliver the actuation signal and process the detection signal. Figure 8 a shows a schematic diagram of the interface electronics. The sensor was operated in resonance mode $(1.77,1.86$ and $1.89 \mathrm{kHz}$ for water, ethanol and white gas, respectively) by running an alternating current through a metal track running over the tube. At the same time two $1.4 \mathrm{MHz}$ signals are fed to the parts of the combs which are attached to the sensor tube. These two signals are in counter-phase to eliminate the influence of parasitic capacitance to the substrate. The counter electrodes are kept at virtual ground by two charge amplifiers (see Figure 8b).

The output signals of the charge amplifiers are amplitude modulated signals of $1.4 \mathrm{MHz}$, where the amplitude is proportional to the sensor capacitance. The signals are demodulated using standard integrated analog multipliers (SA602A) and an op-amp based second-order low pass filter at $3 \mathrm{kHz}$. Summation of the two output signals gives a measure for the difference in capacitance $\left(\mathrm{C}_{1}-\mathrm{C}_{2}\right)$, i.e. the actuation amplitude. The difference between the output signals is a measure for the common variation in the capacitors due to the Coriolis effect. A lock-in amplifier is used to extract the Coriolis signal from the differential output signal in order to eliminate noise and any remaining actuation signal due to nonlinearities.

A syringe pump (CMA Microdialysis CAD system) was used to generate water, ethanol and white gas volume flows between 0 and $1.2 \mathrm{~cm}^{3} / \mathrm{hr}$ through the chip. During the measurement the sensor output signal is recorded, together with the pressure of the liquid using a separate pressure sensor. The pressure sensor signal is proportional to the volume flow rate. It is calibrated at the highest flow level generated by the syringe pump of $1.2 \mathrm{~cm}^{3} / \mathrm{hr}$.

Figure 10 shows the measured output signal as a function of the calculated volume flow (derived from the pressure sensor signal) for water, ethanol and white gas. 


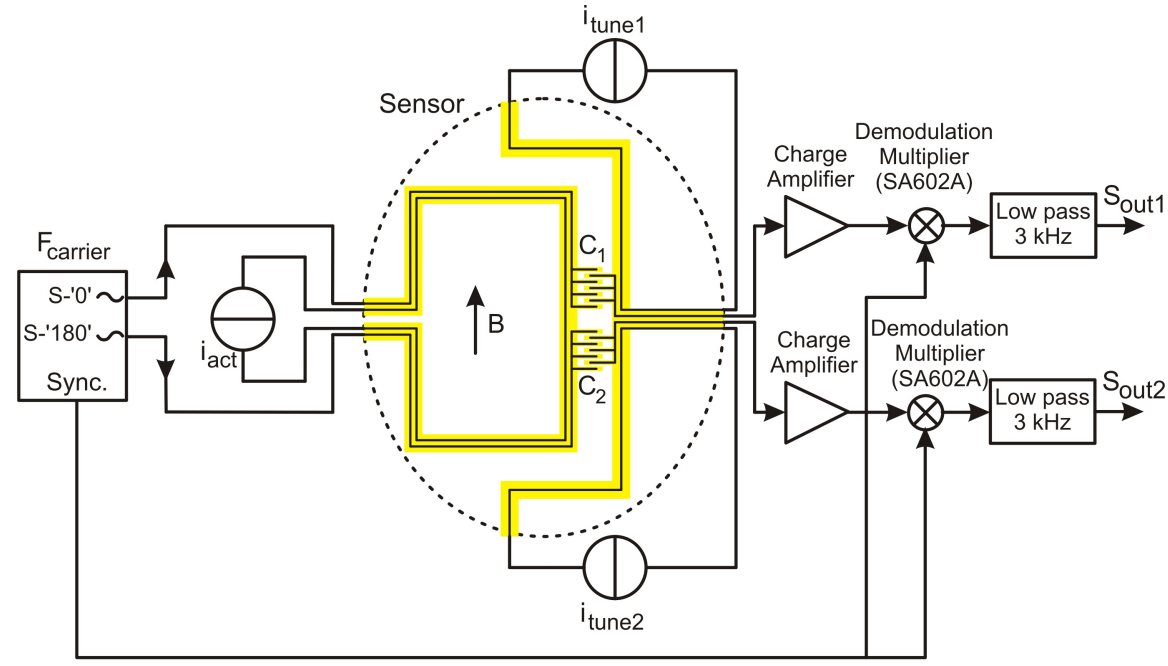

(a)

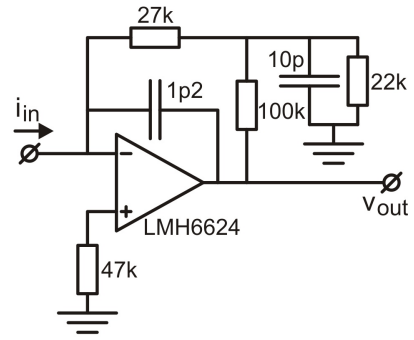

(b)

Figure 8: (a) Schematic diagram of the actuation and readout electronics. (b) Charge amplifier circuit that is used to keep the readout electrodes at virtual ground. The circuit is optimized for carrier frequencies between 1 and $5 \mathrm{MHz}$.

The difference in the slope of the lines is equal to the relative densities of the liquids, indicating that the device directly measures mass flow. Analysis of the measurement data shows that all deviations from the linear fit are less than $2 \%$ of the full scale output signal for each liquid.

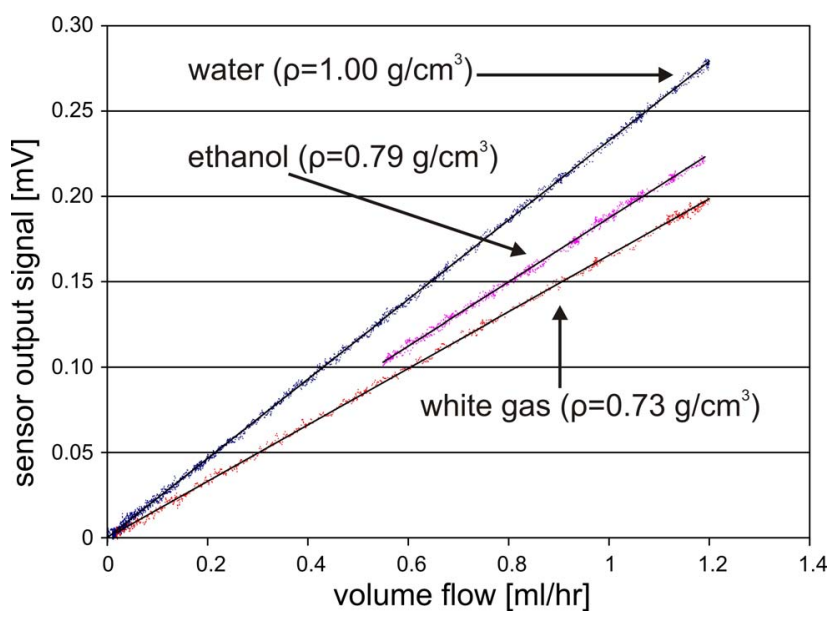

Figure 10: Measured output signal as a function of volume flow for water, ethanol and white gas.

\section{CONCLUSIONS}

We have successfully integrated a capacitive readout structure on a micro Coriolis mass flow sensor chip. The fluidic in- and outlet of the chip are on the back-side of the wafer, allowing for a more stable connection. The sensor shows excellent linear output and accurately measures mass flow in the flow range of $0-1.2 \mathrm{~g} / \mathrm{hr}$. The measurement error is currently in the order of $2 \%$ of the full scale for all measured liquids.

\section{ACKNOWLEDGEMENT}

This research was financed by the Dutch MicroNed program. The authors would like to thank the industrial partners in the project for many fruitful discussions.

\section{REFERENCES}

[1] Y. Mizuno, M. Liger, Y-C. Tai, "Nanofluidic Flowmeter Using Carbon Sensing Element", Proc. MEMS 2004, Maastricht, The Netherlands, Jan. 2225, 2004, p. 322-325.

[2] M. Dijkstra, M.J. de Boer, J.W. Berenschot, T.S.J. Lammerink, R.J. Wiegerink, M. Elwenspoek, "Miniaturized Flow Sensor with Planar Integrated Sensor Structures on Semicircular Surface Channels", Proc. MEMS 2007, Kobe, Japan, Jan. 21-25, 2007, pp. 123-126.

[3] R.C. Baker, "Coriolis Flowmeters: Industrial Practice and Published Information", Flow. Meas. Instrum., Vol. 5, No. 4, pp. 229-246, 1994.

[4] M. Anklin, W. Drahm, A. Rieder, "Coriolis Mass Flowmeters: Overview of the Current State of the Art and Latest Research", Flow. Meas. Instrum., Vol. 17, pp. 317-323, 2006.

[5] A. Mehendale, P.P.L. Regtien, "System Design of Low Capacity Coriolis Mass-flow Meters", $3^{\text {rd }}$ Int. Symp. Sens. Sci., Jülich, Germany, Jul. 18-21, 2005.

[6] J. Haneveld, T.S.J. Lammerink, M. Dijkstra, H. Droogendijk, M.J. de Boer, R.J. Wiegerink, "Highly Sensitive Micro Coriolis Mass Flow Sensor", Proc. MEMS 2008, Tucson, Az, Jan. 13-17, 2008, pp. 920923.

[7] P. Enoksson G. Stemme, E. Stemme, "A Silicon Resonant Sensor Structure for Coriolis Mass-flow Measurements", J. MEMS, Vol. 6, pp. 119-125, 1997.

[8] D. Sparks, R. Smith, J. Cripe, R. Schneider, N. Najafi, "A Portable MEMS Coriolis Mass Flow Sensor", Proc. IEEE Sensors 2003, Toronto, Canada, Oct. 22-24, 2003, pp. 90-92.

[9] M. Dijkstra, M.J. de Boer, J.W. Berenschot, T.S.J. Lammerink, R.J. Wiegerink, M. Elwenspoek, "A Versatile Surface Channel Concept for Microfluidic Applications", JMM, Vol. 17, pp. 1971-1977, 2007.

[10] M.J. de Boer, R.W. Tjerkstra, J.W. Berenschot, H.V. Jansen, G.J. Burger, J.G.E. Gardeniers, M. Elwenspoek, A. van den Berg, "Micromachining of Buried Micro Channels in Silicon", J. MEMS, Vol. 9, No. 1, pp. 94-103, 2000. 